\title{
Fantasías de intervención
}

\author{
ขustavo Bombini / Universidad de Buenos Aires - Universidad Nacional de San Martín \\ gbombini@gmail.com
}

\section{Resumen}

El presente texto realiza un recorrido autobiográfico referido a las relaciones que se van tramando desde la vuelta a la democracia en los años 8o, con la práctica de la escritura en el contexto de la formación inicial en la carrera de Letras y en el desarrollo profesional posterior. Se analizan las condiciones de producción de conocimiento y escritura por parte de profesores e investigadores y se discute sobre el sentido de esta producción en el campo social y político.

Palabras clave: autobiografía • formación en Letras • investigación $\cdot$ transferencia $\cdot$ escritura

\section{Abstract}

This paper draw an autobiographical itinerary about the relations with the writing in the initial formation of professor of Literature and the subsequent professional development since the return of the democracy in the 1980 os. It analyzes the social conditions of production of knowledge and writing by professor and researchers and discusses about the sense of this production in the political and social field.

Key words: autobiography $\cdot$ formation of professors • reserch $\cdot$ transference $\cdot$ writing
Sucedió que como buen estudiante de Letras cualquier relación con la escritura me fue desalentada desde la propia carrera, reducida a la producción de monografías basadas en consignas más o menos erráticas; por otro lado, la escritura de ficción que desde siempre se ha considerado como totalmente divorciada del campo de la formación en Letras, no se presentaba para mí como una potente pulsión de esas que se manifiestan en la adolescencia en la composición precoz de poemas, cuentos o acaso alguna novela catártica.

El pacto con la posibilidad de la escritura se iba a producir de otra manera y, paradójicamente, a partir de la propia academia, o pese a ella. En el pasaje de la academia de la dictadura con su oscurantismo y su mediocridad exasperantes, a la academia de la democracia, la de la "primavera alfonsinista» se produce una trans-
Fecha de recepción: Io/07/2016

Fecha de aceptación: $2 / 08 / 2016$ 
formación en las concepciones acerca de la escritura y fundamentalmente acerca de la posibilidad de su ejercicio. Cierta idea barthesiana flotaba en el ambiente de esa carrera de letras de 2000 ingresantes en 1984 (contra el cupo 200 que ingresábamos con examen de ingreso en la época de la dictadura), "leer es escribir», «la escritura de la lectura», "escribir las lecturas». La idea de que la escritura excedía a la escritura de ficción y que ésta no era la única escritura posible, me provocó un gran alivio. La escritura no era cuestión de talentos innatos, tal la concepción de literatura de la universidad de la dictadura y también de la enseńanza secundaria, y acaso de los medios periodísticos y de otros circuitos artísticos. Una concepción romántica de la literatura y del arte por cierto opresiva que contrastaba con esa concepción telqueliana de la productividad textual que parecía democratizar la posibilidad de pensarse a uno mismo como escritor.

Acaso hayan sido dos textos, de distinta índole, los que marcan el inicio de esta relación poco imaginada pero para toda la vida con la escritura que se empieza a construir apenas un tiempo antes de que terminara de cursar la carrera de Letras de la UBA.

Ciertas notas a pie de página del libro de Josefina Ludmer Onetti. Los procesos de construcción del relato se presentaban como enunciados desafiantes que eran a su vez leídos en el contexto de clases de Seminario de la propia Ludmer donde la escritura se convertía en potente acicate en el proceso de enseñanza:

Tal vez habría que aprender a leer en Latinoamérica, y no solo literatura. Aprender, además, a escribir las lecturas, a poder (abierto el vértigo de la significación) pensar ese vértigo, constituirlo sin que nos enmudezca, ser capaces de contarlo; entregarnos pero sobreponernos; trascenderlo. (...). Leer es un trabajo poético, un trabajo sobre y con las palabras; escribir una lectura puede llegar a ser, meramente, escribir. (1977:I45)

La lectura de esa cita se producía de manera simultánea a la solicitud de escritura desde el Seminario. Recuerdo el temor y ansiedad que me provocó la entrega del primer trabajo de escritura que consistía en el análisis de las concepciones de literatura en textos de Barthes, Benjamin y Freud y que fuera corregido por el profesor Jorge Panesi, quien integraba el equipo del Seminario (Ludmer 2016), con nota 9 (nueve); se trataba de un pequeño triunfo en esa nueva batalla por la escritura que se producía justo en el centro del poder legitimador de la academia emergente apenas recuperada la democracia en los años ochenta.

Por otro lado, las marcas de esa época en mí no terminaron en Ludmer. De manera absolutamente casual, en una librería de Mar del Plata, me topé con el libro Grafein. Teoría y práctica de un taller de escritura (GRAFEIN) cuya autoría colectiva pertenecía al mítico grupo porteño de la década del 70 que por aquellos tiempos, en la cátedra de Noe Jitrik habían leído a Kristeva, a Barthes, a Derrida y desde esa plataforma teórica habían desarrollado una línea de trabajo en talleres, alternativa a la de los talleres literarios a cargo de escritores. La idea de productividad textual, de ese vértigo de la significación de la cita de Ludmer, estaba presente en las entrelíneas de GRAFEIN a lo que se sumaba el componente lúdico y humorísti- 
co, lo que convertía a las consignas de GRAFEIN en acicates estimulantes a prueba de talentos desvalorizados. Podríamos decir que lo que GrafEIN sumaba era cierta impronta didáctica que descansaba en la certeza de que todos podemos escribir. Una verdadera pedagogía de la confianza.

Marcado por la experiencia estudiantil del Seminario Ludmer, instalado acaso ya en el vértigo de la escritura, (Ludmer 2016 y Gerbaudo) y por la lectura reveladora de GRAFEIN sobrevino el primer gran desafío de escritura que fue el de pasar sin estaciones intermedias (salvo unos breves artículos y reseñas en el diario La capital de Mar del Plata) a la publicación de un libro. Aquello que uno haría seguramente con varias décadas de edad avanzada y habiendo leído ToDo, habiendo saldado todas las deudas con la teoría y con la literatura, de pronto - $-\mathrm{y}$ por suerte - se anticipó gracias a la acción temeraria de una gran amiga — que ya no está y a quien mucho extraño-, María Adelia Díaz Rönner. Ella me hizo un pedido acaso en una forma inusual, al menos con respecto a los protocolos de la academia. El pedido fue sencillo: «escribí setenta y cinco páginas sobre esas cosas que me contás que hacés en la secundaria de San Miguel para publicar en "Apuntes”». Se trataba de una colección didáctica que Díaz Rönner dirigía en Libros del Quirquincho, la progresista editorial de literatura infantil creada por Graciela Montes. Fueron dos meses de encierro para escribir esas setenta y cinco páginas en la que claramente se imbricaron las marcas de la formación teórica de la Facultad de Filosofía y Letras (además del «efecto Ludmer» yo me había integrado en esa misma época en la cátedra de Lingüística General, fundamentalmente impactado por los enfoques sociolingüísticos en el estudio del lenguaje que introdujo Beatriz Lavandera) con esas experiencias de profesor primerizo y entusiasta de una escuela secundaria del oeste del Gran Buenos Aires. Esa publicación me marcó, me construyó como sujeto intelectual para el resto de mi trayectoria. Pasé a ser desde julio de 1989 «el autor de La trama de los textos» (Bombini 1989). No se trataba del producto de un proceso de investigación en el sentido estricto del término; se trataba — dado el pedido, dada la colección, dada la editorial — de un trabajo de corte divulgativo dirigido a colegas profesores de nivel secundario. Creo que el «efecto Trama» me cautivó para siempre en la posibilidad/necesidad de escribir para ese colectivo de profesores que enseñan lengua y literatura en la escuela secundaria o que forman profesores; también, de escribir para aquellos y aquellas que se están formando como inminentes profesores sin ir más lejos, en mis cátedras de Didáctica especial en la Universidad de Buenos Aires y de la Universidad Nacional de La Plata. Y en la posibilidad de pensar que esa es una de nuestras formas de producción y circulación de conocimiento en nuestro campo de letras.

Escribir para profesores en formación o para profesores en actividad me llevó a la búsqueda de un registro de escritura que tensionaba seguramente con las exigencias de código que reclama la escritura académica: una escritura en muchos casos opaca, intrincada, tendiente a cierta ilegibilidad que le permite a los miembros de un cierto colectivo profesional institucionalizado (las universidades, el CONICET y otras agencias) sentirse parte de una comunidad altamente distingui- 
da. Pasado el tiempo, comencé a advertir que esa escritura a la que podría llamar de divulgación era en sí misma una forma de producir conocimiento tan legítima y tan compleja como la producción de conocimiento académico. Sin pretender provocar aquí una polémica en torno a esta cuestión que tiene, por ejemplo, en el campo de las ciencias duras su propia versión en lo que se llama divulgación científica o comunicación social de la ciencia, afirmo que hay una discusión pendiente referida al modo en que imaginamos que es posible transmitir algo del orden del conocimiento literario a grandes públicos o a lectores no especializados.

Del conocimiento del libro de GRAFeIn y sobre todo de los años de trabajo compartidos con Maite Alvarado, integrante histórica de ese grupo, quedó una cierta impronta democratizadora que marcó una suerte de credo didáctico a la hora de sostener que todos podemos escribir, que esa práctica no es propia de talentosos creativos o de un mandarinato institucionalizado. El credo es pedagógico y es también social en el sentido de imaginar instancias en las que muchos colectivos sociales escriben, desde los jóvenes que atraviesan cualquier forma de marginalidad hasta los profesores y maestros que habrán de tomar la palabra escrita como memoria de su experiencia docente.

Es en este punto donde las posibilidades de escrituras, propias y ajenas, se diversifican (Bombini 20I2), estallan y superan aquellas oposiciones que se planteaban entre escrituras de ficción, ensayos, escrituras académicas, escrituras sobre la práctica docente, memorias, diarios, escrituras del yo, y acaso, textos hors de gen$r$, textos fuera de género al decir de Régine Robin (8o); seguramente estas nuevas posibilidades de la escritura se producen a contrapelo de la exitosa proliferación de propuestas de lo que hoy llaman alfabetización académica (lo que sería objeto de otro artículo de corte polémico).

Aquello de que leer es también escribir, la potencia inventiva de GRAFEIN y por fin, las investigaciones históricas, sociológicas y antropológicas en relación con la escritura terminaron por convencernos de que los enfoques lingüísticos y normativos puestos en juego por ciertas didácticas dominantes (sobre todo en el campo de la educación superior) acaban empobreciendo la mirada sobre los procesos cognitivos y socioculturales que se ponen en juego en el momento de escribir. En nuestra construcción de una versión socioantropológica o sociocultural de la didáctica de la lengua y la literatura y de los pasajes por la formación de profesores; el tercer factor, diría material, fue el del advenimiento de las nuevas tecnologías (que ya no lo son) y las inéditas posibilidades de circulación de los textos que se escriben. Escribir en la virtualidad parece constituirse en una experiencia de la que todavía poco sabemos, que estaría planteando un nuevo tráfico posible de los textos, nuevas formas de apropiación y además, un nuevo tratado de los géneros de escritura, una retórica flexible y dúctil, abierta a la experimentación. Una experiencia que propone otras reglas que acaso ni siquiera conozcamos y que nosotros mismos estemos creando.

Volviendo a la pregunta del «para quién escribir», hay una inevitable direccionalidad pedagógica en todos nuestros textos, sean de historia literaria o de pedagogía, 
de crítica literaria, de historia o de política cultural. Nuestros textos están ahí porque dan cuenta y rinden cuentas de nuestro modo de construir nuestras trayectorias pero más allá de esto, nos lo propongamos o no, escribir es también escribir para los alumnos, es devenir apunte, fotocopia, ni siquiera libro, mueca de libro y esto conlleva un efecto interesante, al menos por mí experimentado y que tiene que ver con algunas funciones rituales que son propias de la circulación social de la escritura. Me sorprende que en algunos congresos, o espacios de capacitación, en cursos o seminarios a los que soy invitado, sea yo reconocido como el autor de unos textos que la gente lee. Los que están ahí no son mis alumnos de los profesorados universitarios en Letras donde trabajo sino que son alumnos de profesorados universitarios y terciarios de diferentes lugares del país, de ciudades y pueblos del interior del interior, chicos y chicas muy jóvenes que han leído mis textos con mucho interés y que se entusiasman cuando me conocen personalmente y conversamos y me piden tomarse fotos con sus celulares que luego van a parar a sus facebooks y que las atesoran como desafíos a sus docentes formadores que les han recomendado mi bibliografía pero que aún no me conocen; en eso los aventajan a sus profesores.

Estoy seguro de que más allá de esta escena de reconocimiento de la figura de autor facilitada por la tecnología del celular con cámara, lo que se juega ahí es algo del estricto orden de la lectura y del efecto que ciertos textos referidos a la práctica de la enseñanza como los que escribo, provocan en los jóvenes y no tan jóvenes colegas, una sensación de un pacto, de que algo en común nos reúne en esas lecturas/escrituras compartidas, algo que es otra vez del orden de la elección personal: elegir enseñar y en particular, enseñar literatura.

Ese contacto con los lectores y lectoras me ratifica que no se trata de auditorios selectos, ni previsibles, no se trata exactamente de colegas en el sentido de una pequeńa comunidad o cofradía sino de un público extendido y sin duda interesado, que construye la relación con los textos que lee desde un interés relacionado con sus propias prácticas y que reconstruye el ritual de la figura social del autor para reafirmar su interés y su pertenencia a un campo profesional y laboral, el de la enseñanza.

Recuerdo ahora otro artículo de Barthes, leído muy tempranamente y por mi cuenta. Tenía un título atractivo: «Escritores, intelectuales y profesores» (3I3). Lo busco y lo releo ahora mientras escribo y rescato esta cita: "Frente al profesor, que se inclina hacia la palabra, llamaremos escritor a todo operador del lenguaje que se incline hacia la escritura: en medio estaría el intelectual, el que imprime y publica su palabra». Las tres figuras parecen establecer puntos de cruce y articulación y acaso podrían ser cuestionadas en distintos contextos históricos; en todo caso, podría reconocer en mis textos y sobre todo en el modo en que son leídos, el devenir intelectual del profesor en tanto esa palabra oral de la enseñanza se torna escribible, publicable y legible. Por su parte, la pedagogía crítica (Giroux) hablaba del profesor como intelectual y parecía establecer un diálogo con Barthes y con la propia posición profesional que yo estaba buscando adoptar y sobre la que creía que tendría que formar a mis alumnos, futuros profesores. El texto de Barthes parece invitar a pensar en nuevos proyectos de escritura que no son precisamente 
el del anodino paper; quizá lo que esté habilitando sea una nueva tradición en ciernes o recuperando una tradición invisibilizada que vuelve sobre una escritura ausente o desestimada y que acaso mi narrativo libro inicial La trama... ya había venido a recuperar: escribir sobre la propia práctica docente, para narrarla, para comprenderla, para volver y reflexionar sobre ella, para que la práctica se convierta en objeto de la memoria y sea acaso releída e historizada en el futuro.

Y desde aquí vuelvo a la idea planteada antes de una escritura polimorfa, multigenérica y con variadas funciones, con diversos destinatarios y configuradora de una identidad de escritor, profesor, intelectual, como si entre ellas no existieran diferencias: porque la pregunta tiene que ver además con el lugar del estado como garante de la posibilidad de esa escritura. No son los tiempos de los mecenazgos, ni los de la coalición del 80 de fines del siglo xix donde el poder de la política y el de la palabra eran pura zona de intersección. Tampoco son los de la búsqueda de la profesionalización del escritor por la defensa de los derechos de autor y por escritura en la prensa (Payró, Quiroga), sino que los años 80 de fines del siglo $\mathrm{xx}$ son los tiempos del inicio de una nueva relación entre los académicos y el estado (entiéndase la dedicación exclusiva en la universidad, la pertenencia al CONICET) que, por un lado, genera condiciones positivas para la producción de conocimiento (pero a la vez cambiantes según la coyuntura de las políticas de investigación - desde el neoliberalismo desalentador a un proyecto con conciencia nacional estratégica respecto de la producción de conocimiento-) y, por otro lado, parece ponernos a todos bajo unas reglas para la acción y para la escritura (informes, participación en congresos, publicaciones en revistas indexadas, fechas de entrega, carga de datos en sofisticados softwares, informes de rendición de gastos de subsidios y, además, el imperativo de ser citados por otros, bajo las reglas de un tráfico de influencias naturalizado, "citame que cito", "publicame que te publico») que podrían estar constrińendo algo de lo más interesante de nuestra producción y de unos posibles modos de desarrollarla.

Pero la pregunta acuciante, la que siempre le hice y le continúo haciendo a mis alumnos de grado de los profesorados, es la pregunta por lo que yo llamo fantasía de intervención: ¿en qué creemos que modifica, transforma, enriquece el campo disciplinario y el debate público, nuestra producción intelectual?; la pregunta por el sentido, que ha de ser, en definitiva, el deseo de intervención, dispara hacia dos zonas: la de ese lector posible, la de la pregunta de este dossier, «Para quién escribimos» pero también se dispara hacia otra zona, la del estado como garante de nuestras condiciones de producción de escritura y algo así como la deuda contraída de la contraparte; que es lo que nuestras escrituras aportan a un sentido social y político de las cosas; al campo de los saberes y de ahí al resto.

Recuerdo una escena, de cuando yo era becario de la UBA a mediados de los 90 que creo que nos puede ayudar a pensar algo más acerca de la pregunta sobre para quién escribimos. El secretario de Investigación de la Facultad de Filosofía y Letras de la UBA, Félix Schuster, nos citaba un lunes por mes a todos los becarios a reuniones en las que los jóvenes en formación nos hacíamos visibles para 
la institución y para nosotros mismos. En esas reuniones Schuster nos invitaba a presentar nuestros proyectos y avances de investigación (no muchos lo hicieron, yo no) o invitaba a investigadores con trayectoria a reflexionar sobre la práctica de investigación. Recuerdo que en cierto mes de algún año de la segunda mitad de los noventa, la invitada fue Beatriz Sarlo, en ese momento profesora de Literatura Argentina de la Facultad. Sarlo decidió abordar cuestiones que cruzaban los sentidos de las políticas de investigación con los sentidos de nuestra propia posición como investigadores en formación becados por la Universidad. La interpelación fue frontal y causó cierto malestar entre los compañeros. Precisamente, la pregunta fue por el sentido de la investigación, por el impacto social que podría llegar a tener nuestra producción intelectual para lo que argumentó que ese ítem "Transferencia», siempre olvidado al final de los formularios de postulaciones a becas y llenado con una frase de compromiso del tipo «Se realizarán actividades de extensión y/o se publicarán sus resultados» debería ser el primer ítem a llenar por el aspirante a becario. Contaba Sarlo que en esos días, unos especialistas en genética pertenecientes a CONICET habían alcanzado no sé qué resultado respecto de la existencia de vida durante la gestación que permitiría refutar cualquier posición retrógrada de la iglesia respecto de la legalización del aborto.

Existen diversos tiempos para la escritura, para escribirla en secreto o, para ponerla a circular con la convicción de que una escritura ligada con la profesión puede convertirse en una escritura cautivante o, por lo menos, legible, digna de interés o mejor, inmediatamente política, que eso es lo que le suele faltar a nuestras escrituras. Existe un tiempo para pensar en cómo es que se van a poner a escribir los demás y en cómo podemos ser nosotros, los profesores, los mejores habilitadores de la escritura de los otros (y a la vez serlos de la propia).

Por fin, miro con avidez, con interés, con alguna impaciencia, unos proyectos bocetados en sendos cuadernos, dos narraciones posibles, diferentes, una que supone una investigación histórica sobre unos hechos curiosos acaecidos en el Río de La Plata hace doscientos años. Otra, más relacionada con un mundo que conozco bien, el de los profesores y las profesoras, pero sobre todo ellas, las de lengua; siempre transité ese mundo, soy parte; me distancio y lo miro con curiosidad; lo respeto desde que era alumno en la secundaria y así lo sigo mirando; ninguna trayectoria académica me posicionó por arriba de unos sujetos cuya tarea cotidiana es trabajar codo a codo con legiones de pibes que están sumidos en el desinterés y prestos a la curiosidad, todo al mismo tiempo y de manera disruptiva. Ese mundo merece ser narrado, ese mundo lleno de matices, de afectos, de códigos internos, de guiños, de saberes específicos, de tareas cotidianas, de rutinas, de frustraciones, de sueños... y de fantasías de intervención.

\section{Bibliografía}

Barthes, Roland (1986). Lo obvio y lo obtuso. Buenos Aires: Paidós.

Bombini, Gustavo (1989 [2005]). La trama de los textos. Problemas de la enseñanza de la literatura.

Buenos Aires: Lugar Editorial. 
(Comp.) (20I2). Escribir la metamorfosis. Escritura y formación docente. Buenos Aires: El Hacedor.

Gerbaudo, Analía (2016). Politicas de exhumación. Las clases de los críticos en la universidad argentina de la posdictadura (1984-1986). Santa Fe: Universidad Nacional de General Sarmiento/Universidad Nacional del Litoral.

Giroux, Henry (1990). Los profesores como intelectual. Hacia una teoría critica del aprendizaje. Barcelona: Paidós.

GRAFEIN (198I). Grafein. Teoría y práctica de un taller de escritura. Madrid: Altalena.

Ludmer, Josefina (1977). Onetti. Los procesos de construcción del relato. Buenos Aires: Sudamericana.

(2016). Clases 1985. Algunos problemas de teoría literaria. Edición y prólogo Annick Louis.

RobIN, RéGINe (I996). Identidad, memoria y relato. La imposible narración de sí mismo. Buenos Aires: Свс/Universidad de Buenos Aires. 